the amino-terminal side, the third loop from the loop/helix cap is exposed on the surface (Fig. 1). The authors propose that this loop is critical in mediating the formation of a hexameric complex between Smad4 trimers and trimers of phosphorylated, receptorregulated SMADs.

If TGF- $\beta$-mediated signalling occurs through the ligand-dependent assembly of SMAD hetero-oligomers, how is the formation of these complexes regulated? Hata et al. provide biochemical evidence that the amino-terminal domain of either Smad2 or Smad4 physically interacts with the carboxyterminal domain, to prevent the formation of a Smad2-Smad4 complex. Although several models are consistent with these data, the amino-terminal domain could, perhaps, fold back on the carboxy-terminal domain to control its activity, possibly by altering the conformation or sterically hindering the accessibility of the third loop (Fig. 1).

Most of the mutations that affect Madrelated genes in cancer cells or in invertebrate development map to the carboxy-terminal domain. Based on structural and functional criteria, Shi et al. have now defined three types of substitutions that affect SMAD function. The first class of mutations cause amino-acid substitutions that map to the interface regions between the Smad4 monomers, thereby destabilizing the trimeric complex. The second class map to the third loop on the face of the disk, and these mutations may disrupt the formation of heteromeric complexes. The third class map to the hydrophobic core, and such mutations may destabilize the structure of the protein. But mutant proteins from any of these classes will disrupt the formation of hexameric complexes - so, presumably, they block the transmission of a TGF- $\beta$ signal.

In contrast to the carboxy-terminal domain, relatively few of the mutations that are associated with a cancerous phenotype have been mapped to the amino-terminal domains of MAD-related proteins. However, two mutations that affect the same amino-terminal arginine residue have been found in both Smad 2 and Smad4. Hata et al. have characterized these mutations, and they show that they may increase the affinity of the amino-terminal domain for the carboxy terminus. In essence, this locks the regulatory domain onto the effector domain and so prevents signalling of events that would presumably lead to the suppression of cell growth. Such a gain of autoinhibitory activity would represent an unusual mechanism by which to inactivate a tumour suppressor, and it could explain why relatively few missense mutations have been identified in the amino-terminal region of SMADs.

Smad4 does not possess carboxy-terminal serine residues, and it is not regulated by phosphorylation. Yet serine phosphorylation is critical to the function of Smads 1, 2, 3 and 5. So what structural role does phosphorylation play in controlling the function of these receptor-regulated SMADs? By analogy with the Smad4 structure, the carboxy-terminal serines of these SMADs are likely to be accessible to the type-I receptor serine/threonine kinase. Phosphorylation at the tail could thus relieve autoinhibition by the amino-terminal domain, revealing the third loop and allowing the formation of hetero-oligomers (Fig. 1).

Interestingly, many of the mutations characterized within the context of the Smad4 structure were originally identified in receptor-regulated SMADs, and they interfere with their phosphorylation ${ }^{4,9}$. Because phosphorylation is required for the formation of Smad2-Smad 4 complexes, the main defect in these mutants could be in phosphorylation, in the interaction with Smad4, or a combination of both. Solving the structure of full-length SMAD proteins should provide some answers, as well as insights into how the structure of these signalling molecules regulates their function. But remaining issues include the molecular basis for recognition of specific SMADs by individual receptors, the basis for nuclear accumulation of phosphorylated SMADs, and the precise mechanisms by which SMADs control gene expression.

Jeff Wrana is in the Program in Developmental Biology and Division of Gastroenterology, The Hospital for Sick Children, Toronto, Ontario, Canada M5G 1X8. Tony Pawson is in the Program in Molecular Biology and Cancer, Samuel Lunenfeld Research Institute, Mount Sinai Hospital, Toronto,

\section{Ontario, Canada M5G 1 X8.}

1. Hata, A., Lo, R. S., Wotton, D., Lagna, G. \& Massagué, J. Nature 388, 82-87 (1997).

2. Shi, Y., Hata, A., Lo, R. S., Massagué, J. \& Pavletich, N. P. Nature 388, 87-93 (1997).

3. Hahn, S. A. et al. Science 271, 350-353 (1996).

4. Eppert, K. et al. Cell 86, 543-552 (1996).

5. Zhang, Y., Feng, X.-H., Wu, R.-Y. \& Derynck, R. Nature 383, 168-172 (1996)

6. Lagna, G., Hata, A., Hemmati-Brivanlou, A. \& Massagué, J. Nature 383, 832-836 (1996).

7. Macías-Silva, M. et al. Cell 87, 1215-1224 (1996).

8. Chen, X., Rubock, M. J. \& Whitman, M. Nature 383, 691-696 (1996).

9. Hoodless, P. A. et al. Cell 85, 489-500 (1996).

Semiconductors

\title{
Do the twist to get fit
}

Pauline Rigby

T he substrate problem is a big one for the optoelectronics industry. It is only possible to grow good-quality material, without any defects that would prevent light emission, if a substrate can be found with an almost identical atomic spacing to the layer grown on it. This is lattice matching. For some material systems, such as gallium nitride $(\mathrm{GaN})$, there is no lattice-matched substrate available, so it proved extremely difficult to grow defect-free material, and the development of $\mathrm{GaN}$ for blue light emission was delayed for 20 years. To magnify the problem, dozens of different materials are required to cover the wavelength ranges of all optoelectronics applications, and each needs a latticematched substrate. But now Ejeckam et al. ${ }^{1}$ have invented a substrate that doesn't require lattice matching - a universal substrate.

When an epitaxial layer is grown on a substrate with a different lattice parameter, at first the growing layer elastically distorts itself to fit the crystal matrix so that the lattice planes are continuous across the interface. When a certain critical thickness ${ }^{2}$ is exceeded, the elastic energy is released by plastic deformation, in the form of dislocations which nucleate at the surface and then travel in to the interface. A dislocation is a boundary between part of a crystal that has slipped and part that hasn't (Fig. 1). As it moves through the crystal it displaces one row of atoms at a time; on reaching the far side it has displaced an entire layer. The energy barrier to this process is much lower than it would be

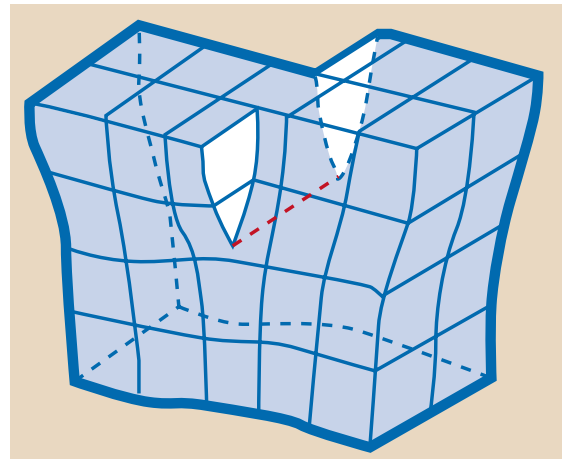

Figure 1 A screw dislocation in a simple cubic lattice.

for all the rows of atoms to move at once.

One approach to the problem is to make the substrate very thin, so extending the critical thickness by sharing the strain between the substrate and overlayer ${ }^{3}$. As one side of the structure is in tension and the other in compression, the structure bends like a bimetallic strip. These structures are extremely fragile, too fragile to withstand normal wafer handling and processing.

Dislocations are usually detrimental to device performance, but Ejeckam et al. have put them to work, to make a stretchy universal substrate. A film of gallium arsenide (GaAs) only $30 \AA$ thick is grown on an ordinary GaAs substrate (with an aluminium arsenide (AlAs) spacer layer in between), then the thin film is bonded to another substrate, essentially by pressing the surfaces 


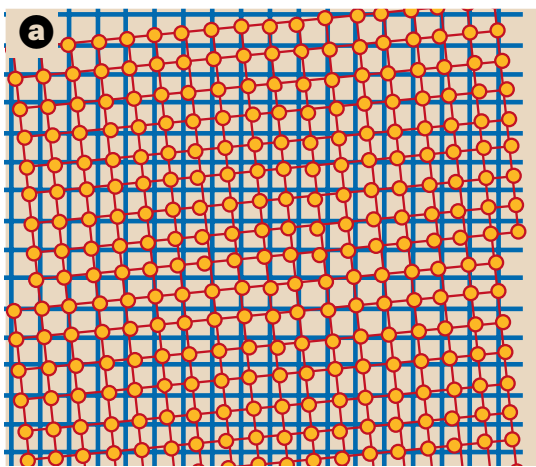

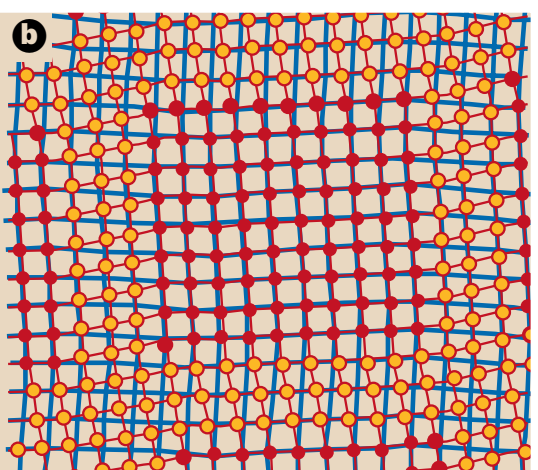

Figure 2 Formation of a twist boundary by rotation about an axis perpendicular to the page. Circles are atoms just above the boundary, and the blue grid marks those below. a, The atom positions resulting from a rigid rotation. Very few of the atom positions in upper and lower parts of the crystal are coincident. But a cross-grid of screw dislocations, b, permits regions of good match (red) to remain for the same angle of rotation. Around the dislocations the atoms are relatively free to move because the bonds are stretched and therefore weaker. As the angle of twist is increased, more dislocations are needed to accommodate the mismatch, forcing the dislocations closer together and shrinking the regions of good match, so that more of the surface becomes elastic. A layer of another material, with a different lattice constant, can then be grown on top, and the substrate stretches to accommodate it.

together at high temperature. The surfaces must be atomically smooth and chemically cleaned so that the atomic bonds are reformed. After fusing the surfaces together, the original GaAs substrate and the AlAs are removed with selective chemical etches.

What makes the universal substrate work is a twist boundary ${ }^{4}$. This is created by rotating the thin film before fusing it to the substrate; in crystallographic terms, introducing an angular misalignment between the <011> directions of the two lattices. A grid of screw dislocations (Fig. 1) exists in the plane of the interface (Fig. 2) to accommodate the twist. At the cores of the dislocations the atomic bonds are stretched. The final thin film is stretchy rather than rigid because it is sitting on stretched, weakened bonds, and so it puts less stress on any mismatched layer grown on it.

Because the dislocations are fully contained at the interface and don't thread through the thickness of the layer, they don't interfere with the light-emitting properties of the material. To prove the concept, Ejeckam et al. first grew In GaP on a universal substrate ${ }^{1}$. The lattice mismatch was $1 \%$, yet they were able to grow a layer $3,000 \AA$ thick without defects - 30 times the critical thickness.

Then, getting bolder, they tried growing gallium antimonide $(\mathrm{GaSb})$. The lattice constant of GaSb is $6.096 \AA$, whereas that of GaAs is $5.653 \AA$ - a strain of $8 \%$. Under ordinary conditions defects would start to invade the perfect crystal after four or five monolayers (single-atom layers) had been grown. But the 3,000 A layer of GaSb grown on a universal substrate is defect-free, despite being more than 300 times the critical thickness (as they reported at a meeting in $\mathrm{May}^{\star}$ ).

A universal substrate made of silicon would be attractive because silicon is the most abundant natural semiconductor, and con-

* Conference on Lasers and Electro-optics, Baltimore, MD, USA, 18-23 May 1997. ducts electricity well. GaAs is the most widely used synthetic semiconductor in the world and it is also an efficient light emitter, unlike silicon, but it has poor conductivity. Using the new technology, it should be possible to combine the best features of both materials on a single optoelectronic chip. Such a chip could use photons to transmit information, while at the same time performing conventional electronic tasks, such as the logic functions that form the basis of computing.

How about a material that is intrinsically capable of both functions? Indium antimonide (InSb) may be such a material ${ }^{5}$ : it is luminescent, and has electron and hole mobilities many times better than silicon. The lattice mismatch between InSb and GaAs is $15 \%$, and its critical thickness is less than a monolayer. Nevertheless, a 6,500- $\AA$ thick, dislocation-free layer of InSb has now been grown for the first time, on a universal substrate ${ }^{6}$.

The universal substrate needs to be tested further to determine its limits, but so far it has surpassed the wildest expectations, and has drawn the attention of many leading semiconductor-device manufacturers. The twist principle isn't limited to one type of material, so it could lead to the realization of new classes of compounds, even beyond the realm of semiconductors.

Pauline Rigby is in the Department of Engineering Science, Oxford University, Parks Road, Oxford

OX1 3PJ, UK

e-mail:pauline.rigby@eng.ox.ac.uk

. Ejeckam, F. E., Lo, Y. H., Subramanian, S., Hou, H. Q. \&

Hammons, B. E. Appl. Phys. Lett. 70, 1685-1687 (1997)

Mathews, J. W. \& Blakeslee, A. E. J. Cryst. Growth 27, 118-125

(1974)

Lo, Y. H. Appl. Phys. Lett. 59, 2311-2313 (1991).

4. Hull, D. \& Bacon, D. J. Introduction to Dislocations 3rd edn, 143-144 (Pergamon, Oxford, 1984).

Biefeld, R. M. \& Hebner, G. A. Appl. Phys. Lett. 57, 1563-1565 (1990)

6. Ejeckam, F. E., Lo, Y. H., Seaford, M. L., Hou, H. Q. \& Hammons, B. E. Appl. Phys. Lett. (in the press).

\section{Daedalus}

The watch on the line

Computer database technology is slowly building a subtle tyranny. Already each of us is pinned down by myriad financial and personal records. Security cameras now pose a new threat. Formally, they exist to counter crime. But the growing power of pattern-recognition software will soon allow them to identify and remember the faces and body styles of single individuals. Camera networks will then be able to track every citizen all the time. One possible defence is disguise. False noses, eye patches, stick-on moustaches, joke protruding ears, surgical boots and so on, will become de rigueur for all public appearances.

Such dodges, says Daedalus, evade the real issue, which is not surveillance but secrecy. The faceless authorities behind the cameras know all about us; we know nothing about them. Freedom of video information seems a better option. Secrecy would vanish; so would privacy, of course.

In a sense, this is the ultimate logic of the electronic 'global village'. A traditional local village had no privacy; gossip made sure of that. The result was a very low rate of crime and deviancy, and strong social cohesion. The same democracy without privacy might work well for the global village. In this connection, Daedalus recalls his scheme of last week, for coding video signals for easy Internet transmission. So he suggests connecting all security cameras to the Internet.

Crime would plummet. A global audience of eager voyeurs would be far more vigilant than the tired minions who now gaze at the screens. Indeed, many would have a keen personal interest. Motorists would keep an eye on their own cars, parents would track their own children and wives their own husbands, and any criminal caught in the act would probably be recognized by somebody. But profounder consequences could follow. For a start, word of any event worth watching would spread rapidly on the Net. So TV news would be rescued from the selection, editing and slanting of the professionals. And a new form of live entertainment would be born, displacing the endless boring channels of artificial entertainment. Who would bother with a chat show or a soap opera when real crime, sex, disaster, exhibitionism and embarrassment were on offer? And anyone could put on a spontaneous global entertainment anywhere at any time, courtesy of their friendly neighbourhood security camera. Warhol's prediction would come true: we could all be famous for 15 minutes.

David Jones 